\title{
Variational modularity at the cell level: insights from the sperm head of the house mouse
}

\author{
Nuria Medarde ${ }^{1 *}$, Francesc Muñoz-Muñoz ${ }^{1}$, María José López-Fuster ${ }^{2}$ and Jacint Ventura ${ }^{1 *}$
}

\begin{abstract}
Background: Modularity is an important feature in the evolvability of organisms, since it allows the occurrence of complex adaptations at every single level of biological systems. While at the cellular level the modular organization of molecular interactions has been analyzed in detail, the phenotypic modularity (or variational modularity) of cell shape remains unexplored. The mammalian spermatozoon constitutes one of the most complex and specialized cell types found in organisms. The structural heterogeneity found in the sperm head suggests an association between its inner composition, shape and specificity of function. However, little is known about the extent of the connections between these features. Taking advantage of the house mouse sperm morphology, we analyzed the variational modularity of the sperm head by testing several hypotheses related to its structural and functional organization. Because chromosomal rearrangements can affect the genotype-phenotype map of individuals and thus modify the patterns of covariation between traits, we also evaluate the effect of Robertsonian translocations on the modularity pattern of the sperm head.

Results: The results indicated that the house mouse sperm head is divided into three variational modules (the acrosomal, post-acrosomal and ventral spur module), which correspond to the main regions of the cytoskeletal mesh beneath the plasma membrane, i.e., the perinuclear theca. Most of the covariation is concentrated between the ventral spur and the acrosomal and post-acrosomal modules. Although the Rb fusions did not alter the main modularity pattern, they did affect the percentages of covariation between pairs of modules.

Conclusions: The structural heterogeneity of the cytoskeleton is responsible for the modular organization of the sperm head shape, corroborating the role that this structure has in maintaining the cell shape. The reduction in percentages of shape covariation between pairs of modules in Rb sperms suggests that chromosomal rearrangements could induce changes in the genotype-phenotype map. Nevertheless, how these variations affect sperm fertilization success is yet to be elucidated.
\end{abstract}

Keywords: Variational modularity, Sperm morphology, Geometric morphometrics, Mus musculus domesticus, Robertsonian system

\section{Background}

Organisms are composed of elements that, although coordinated, show obvious signs of heterogeneity with respect to certain kinds of processes [1,2]. These elements, called modules, are internally integrated but relatively independent of one another [2]. Thus, modularity is considered a key feature of biological organization that allows the modification of certain parts of organisms

\footnotetext{
*Correspondence: nuriaestel.medarde@uab.cat; jacint.ventura.queija@uab.cat ${ }^{1}$ Departament de Biologia Animal, Biologia Vegetal i Ecologia, Facultat de Biociències, Universitat Autònoma de Barcelona, Cerdanyola del Vallès 08193, Spain

Full list of author information is available at the end of the article
}

with minor effects on other parts, thereby contributing to evolvability [3]. Modularity occurs at every single level of biological organization, from molecular interactions to networks of ecological connections [1,2]. Variational modularity (that is, groups of correlated characters) has long been recognized in morphological traits $[1,2]$ since it provides the evolutionary flexibility required to induce adaptive changes in certain regions of complex phenotypic structures. At the cell level, the structural and functional modularity of molecular networks have been studied in detail $[4,5]$, but to our knowledge, the variational modularity of cell morphology has not been examined to date. The relations between different kinds 
of modularity in biological organization are still not well understood, and their comparative study may provide insights into evolutionary processes [6]. The male gametes of certain mammals may represent an ideal model for testing the connections between different kinds of modularity, as they are highly polarized cells with structurally and functionally differentiated regions that are morphologically recognizable [7].

The sperm of the western house mouse, Mus musculus domesticus, has a complex, flattened, hook-shaped head containing the cell nucleus [7]. Overlying the nucleus is the acrosome, which has two functional components: the anterior acrosome and the equatorial segment (Figure 1) $[7,8]$. Division at the organelle level also affects the organization of the plasma membrane and the cytoskeleton. The plasmalemma of the sperm head is structurally and functionally differentiated into two major domains, the acrosomal and the post-acrosomal plasma membranes, separated by the sub-acrosomal ring $[8,9]$. Beneath the plasma membrane there is a dense cytoskeletal mesh that forms the perinuclear theca (PT), which is also differentiated into two main domains: the outer periacrosomal layer and the post-acrosomal sheath $[10,11]$. In the latter domain, an area mainly composed by perforatorial proteins is distinguished: the ventral spur region $[10,11]$. In view of this noticeable compartmentation in the sperm head, the main goal of this study is to assess, for the first time, whether the structural and functional regionalization of the cellular components generates the variational modularity of cell shape. We used geometric morphometrics to test three hypotheses that divide the sperm head into different modules (Figure 1): i) acrosome and post-acrosome (H1); ii) anterior acrosome, equatorial segment and post-acrosome (H2); and iii) acrosome, postacrosome and ventral spur (H3).

Previous studies in mice from the Robertsonian system found in Barcelona (BRbS) revealed that chromosomal rearrangements affect the size and the shape of the sperm head [12]. This $\mathrm{Rb}$ system represents a unique contact zone between standard (St) and $\mathrm{Rb}$ mice since there is no evidence of a $\mathrm{Rb}$ race in which a group of individuals from the same geographical area share a set of metacentrics in homozygous condition [13]. Diploid numbers range from 27 to 40 chromosomes, and seven different metacentrics (Rb3.8, 4.14, 5.15, 6.10, 7.17, 9.11 and 12.13) have been described up to now [14-16]. The relative stability of its metacentric staggered structure [16] as well as the phenotypic differences associated with karyotype detected in animals from this area [17] suggest the presence of partial barriers to gene flow. In this scenario, the study of the factors involved in the establishment of reproductive barriers between individuals may take on special relevance. Thus, assuming that $\mathrm{Rb}$ fusions could induce variations in the genotype-phenotype map of the sperm head [12], and that changes in the variational modularity patterns may play an important role in the evolvability of the sperm features, a second aim of this study is to evaluate the effect, if any, of the Rb translocations on the pattern of variational modularity of the western house mouse sperm head.

\section{Results and discussion}

The Procrustes ANOVA performed on the replicated subsample showed highly significant differences between sperm heads (MS sperms $=0.000388$, MS error $=0.000001$, $P<0.0001)$. The mean squares for sperm head variation
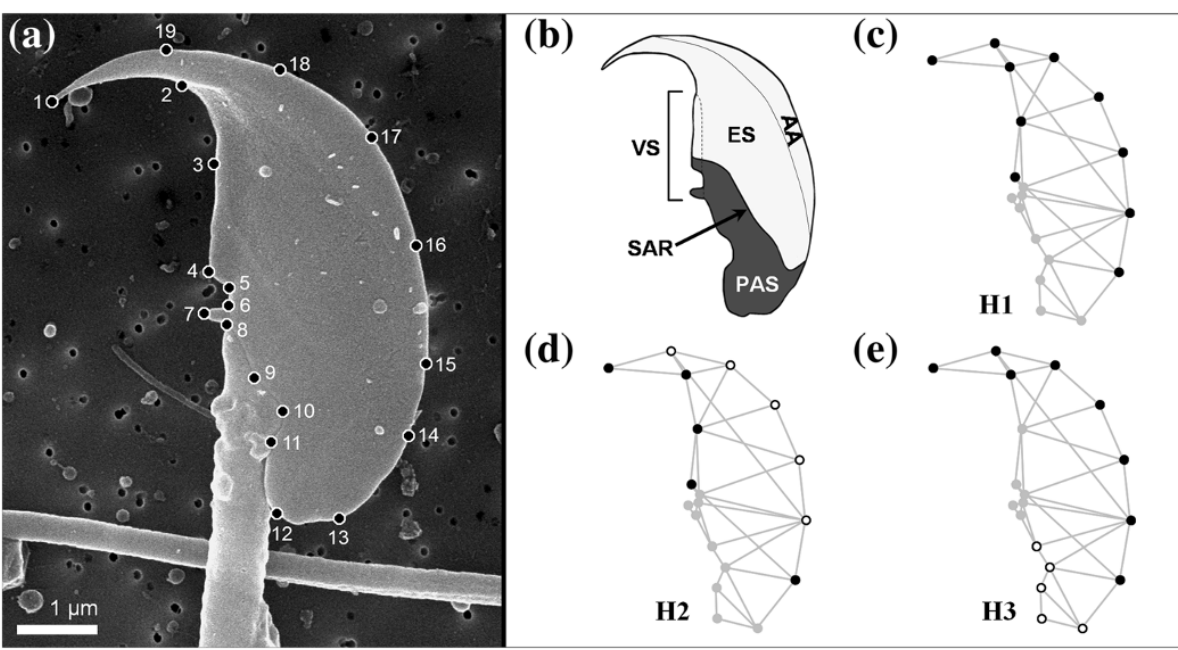

Figure 1 House mouse sperm head. (a) SEM image of the sperm head indicating the position of landmarks and semilandmarks. (b) Localization of the main regions of the sperm head: AA, anterior acrosome; ES, equatorial segment; SAR, sub-acrosomic ring; PAS, post-acrosomal sheath; VS, ventral spur. The acrosomal (APM) and post-acrosomal (PAPM) plasma membranes are shown in light and dark shading respectively. (c-e) Subsets of landmarks for hypotheses $\mathrm{H} 1-\mathrm{H} 3$. 
exceeded the mean squares for replicates by 388 -fold, indicating low measurement error and consequently strong repeatability of the landmark location in the sperm head.

Fine morphological analysis of mouse sperm heads combining scanning electron microscopy and geometric morphometrics revealed significant allometry of cell shape $(P<0.001)$ with $11.8 \%$ and $8.6 \%$ of shape variation explained by changes in cell size in the St and Rb groups, respectively. Allometric shape changes affected all the landmarks to a similar degree and mainly involved a narrowing of the sperm head and a stretching of the hook. The existence of significant size-dependent shape changes is interesting because evidence of shape allometry at the cell level is very scarce. The precise mechanisms that underlie this association are unknown. However, recent studies have indicated a correlation between cell shape and growth $[18,19]$, and between cell size and the behaviour of the cytoskeletal machinery [19]. Given that the cytoskeleton is mainly responsible for shaping the cell during growth, it is reasonable to suppose that these behavioural changes in the cytoskeletal machinery may be partly responsible for the association between size and shape of the cell. Because allometry represents a global integration factor, the residuals of the multivariate regression of the Procrustes coordinates onto log CS were used for further analyses.

The PCA indicated that the first five PCs explained around $75 \%$ of the shape variation in both $\mathrm{St}$ and $\mathrm{Rb}$ mice (Figure 2; Table 1). The shape changes associated with PC1 were mainly concentrated in the hook and the convex side of the sperm head, while the changes associated with $\mathrm{PC} 2$ affected the ventral spur and postacrosome (see Figure 2). The $R V$ and multiset $R V$ coefficients indicated that there was a low to moderate degree of covariation between the different regions of the sperm head (Figure 3). However, the only significant hypothesis when comparing the multiset $R V$ value with permutational distributions was $\mathrm{H} 3$, which divides the sperm head in accordance with the structural division of the PT (Figure 3). The PT is a cytoskeletal structure with a central role in the morphogenesis and maintenance of sperm head shape [20,21]. While its heterogeneous composition is associated with the functional organization of the cell and the subdivision of the plasmalemma [11], our results indicate that the modularity of the sperm head shape is directly influenced by structural changes in the cytoskeleton. The functional division of the sperm head into three main regions (H2) seems not to play a direct role in the variational modularity of overall shape.

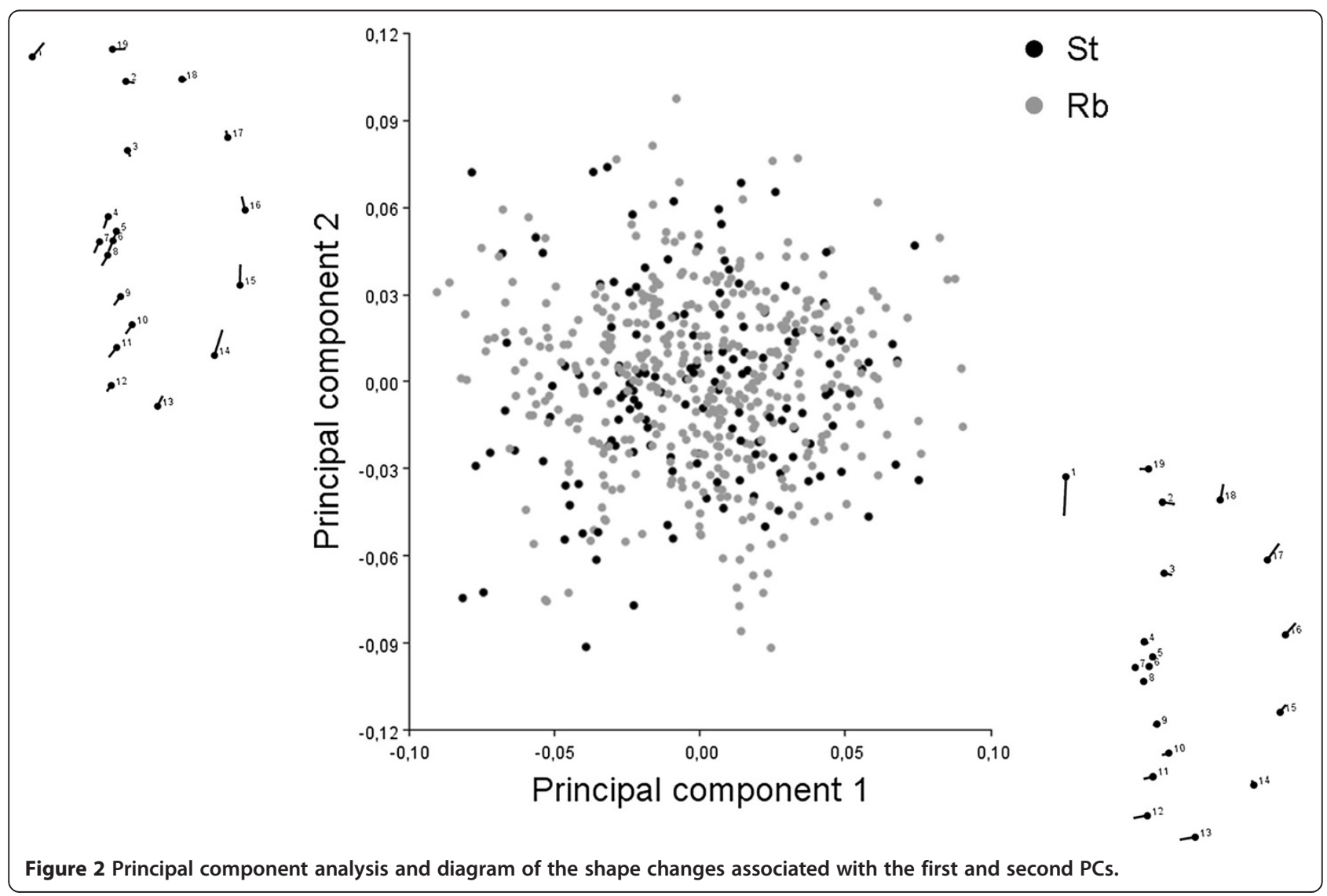


Table 1 Eigenvalues and percentages of variance and cumulative variance explained by the first ten principal components (out of 34) of the PCA obtained with the residuals from the multivariate regression analysis

\begin{tabular}{|c|c|c|c|c|c|c|}
\hline \multirow[b]{2}{*}{ PC } & \multicolumn{3}{|c|}{ St } & \multicolumn{3}{|c|}{$\mathbf{R b}$} \\
\hline & Eigenvalues & Variance (\%) & Cumulative (\%) & Eigenvalues & Variance (\%) & Cumulative (\%) \\
\hline 1. & 0.00144 & 25.155 & 25.155 & 0.00147 & 27.376 & 27.376 \\
\hline 2. & 0.00107 & 18.594 & 43.749 & 0.00090 & 16.700 & 44.076 \\
\hline 3. & 0.00076 & 13.238 & 56.987 & 0.00067 & 12.518 & 56.594 \\
\hline 4. & 0.00056 & 9.731 & 66.718 & 0.00055 & 10.288 & 66.883 \\
\hline 5. & 0.00046 & 8.008 & 74.726 & 0.00042 & 7.823 & 74.706 \\
\hline 6. & 0.00027 & 4.708 & 79.434 & 0.00025 & 4.701 & 79.407 \\
\hline 7. & 0.00022 & 3.907 & 83.341 & 0.00023 & 4.306 & 83.713 \\
\hline 8. & 0.00019 & 3.371 & 86.711 & 0.00018 & 3.419 & 87.132 \\
\hline 9. & 0.00015 & 2.629 & 89.341 & 0.00013 & 2.431 & 89.563 \\
\hline 10. & 0.00014 & 2.383 & 91.723 & 0.00012 & 2.205 & 91.768 \\
\hline
\end{tabular}

Abbreviations: PC, Principal components; St, standard sperm heads; $R b$, Robertsonian sperm heads.

Moreover, according to our results, the acrosome behaves as an integrated unit, while in the post-acrosomal region the ventral spur shows a high degree of autonomy. This modularity pattern was detected in data corrected and not corrected (results not shown) for allometry. This result indicates that size-dependent shape changes do not play a major role in the patterns of integration of the sperm head. The $R V$ values obtained in the 2B-PLS analyses indicated a low strength of association between subsets of landmarks in $\mathrm{H} 3$ for all comparisons (Table 2). However, the percentages of covariation explained by the first PLS axis in the comparisons of the ventral spurs with the acrosomal and post-acrosomal domains were high, especially in the St group (Table 2). This

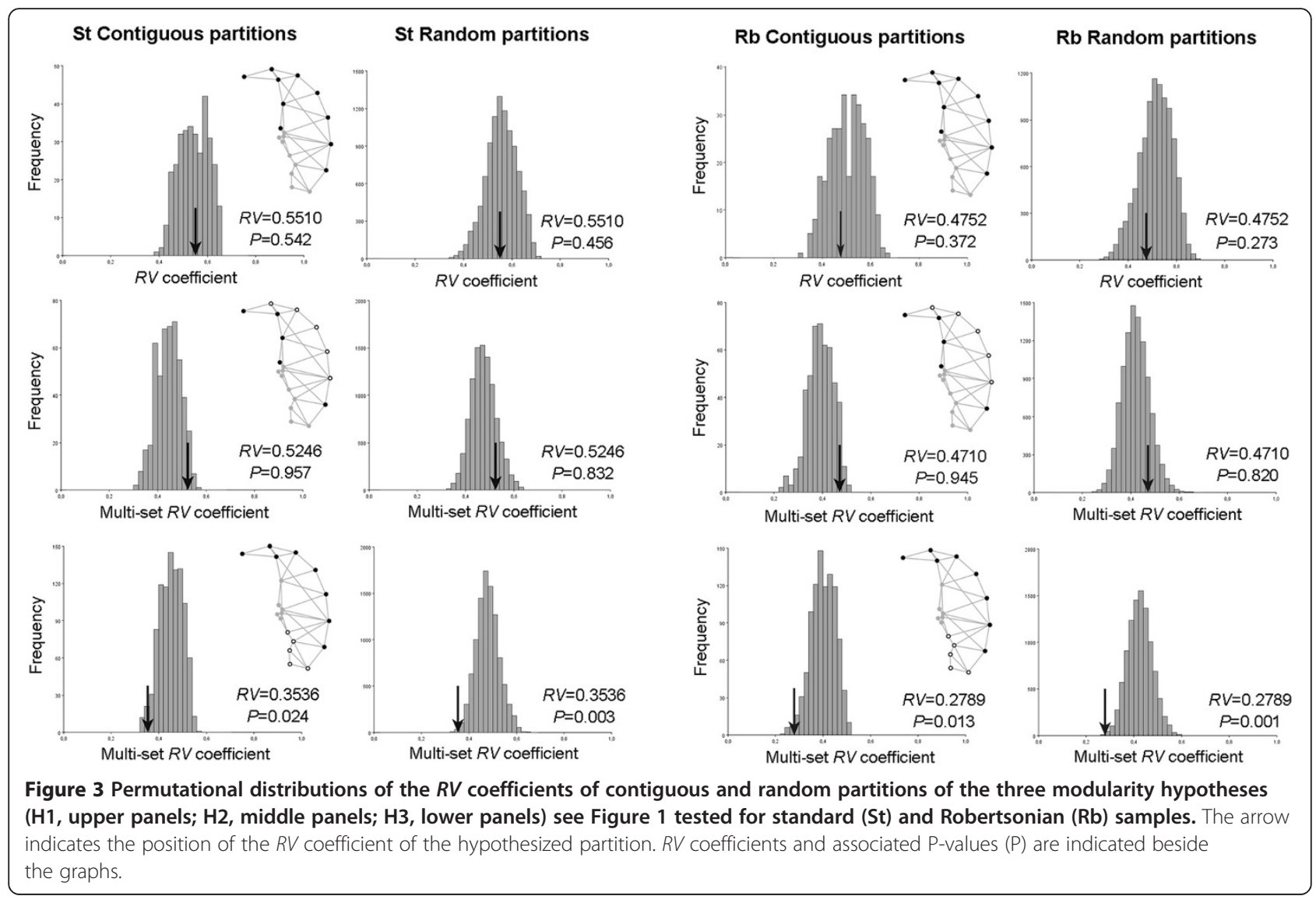


Table 2 Results of 2B-PLS analyses for standard (St) and Robertsonian (Rb) samples

\begin{tabular}{clcccrc}
\hline Group & Blocks & $\boldsymbol{R V}$ & $\mathbf{P}$ & \%Total Cov PLS1 & Corr PLS1 & P \\
\hline \multirow{3}{*}{ St } & AC vs PA & 0.034 & 0.0071 & 61.8 & 0.282 & 0.0001 \\
& AC vs VS & 0.035 & 0.0064 & 86.3 & 0.330 & 0.0001 \\
& PA vs VS & 0.067 & 0.0001 & 83.7 & 0.321 & 0.0001 \\
\multirow{2}{*}{ Rb } & AC vs PA & 0.045 & 0.0001 & 55.5 & 0.307 & 0.0022 \\
& AC vs VS & 0.027 & 0.0043 & 56.9 & 0.336 & 0.0001 \\
& PA vs VS & 0.031 & 0.0001 & 52.2 & 0.0001 \\
\hline
\end{tabular}

For each two-block comparison we display the $R V$ coefficient; the percentage of total covariation explained by the first PLS axis; the correlation between the 2B-PLS1 scores; and the associated P-values (P).

result is congruent since the ventral spurs are considered to be specialized features integrated in the post-acrosomal sheath $[10,20]$. The high percentages of covariance explained by the first PLS axis in the St sperm head indicated that most of the covariation is concentrated in precise features of shape that change in a coordinated manner between modules. This is especially relevant in the comparison between the ventral spur and the acrosomal and post-acrosomal modules, where the first PLS explains around $85 \%$ of the covariation. These results suggest that the amount of covariation between certain pairs of modules may depend on the subdivision of the sperm plasma membrane, since the acrosomal domain overlies the whole acrosome and the upper ventral spur and the post-acrosomal domain involves the post-acrosome and the lower region of the ventral spur module $[8,9]$. In fact, the hypothesis testing the division of the sperm head into acrosomal and post-acrosomal modules (H1), although not significant, yielded an $R V$ value lower than most of the alternative partitions, indicating a certain influence of the membrane domains. Conversely, the 2BPLS revealed a different covariation pattern in $\mathrm{Rb}$ sperm heads. Several studies have evidenced that chromosomal rearrangements may induce changes in morphological covariation patterns through the rupture of genetic linkage groups and/or the occurrence of epistatic interactions between genes involved in the development of certain modules [22,23]. Under these circumstances, the variation in the genotype-phenotype map could explain the lower percentages of covariation among pairs of modules detected in $\mathrm{Rb}$ mice. However, the extent to which these differences affect the potential for evolutionary change remains to be elucidated.

\section{Conclusions}

Our results reveal for first time the existence of variational modularity in a cellular structure such as the house mouse sperm head and highlight the important role of the cytoskeleton in maintaining the shape of the cell. The presence of $\mathrm{Rb}$ translocations did not affect the variational modularity pattern. However, the lower percentages of shape covariation between pairs of modules in $\mathrm{Rb}$ sperms heads suggest a certain influence of the $\mathrm{Rb}$ rearrangements. Understanding the mechanisms that alter covariation between phenotypic traits in the sperm head is an aspect of great importance given its possible effect on the evolvability of these specialized cells. However, the extent to which these changes affect sperm fertilization success is a subject for further studies.

\section{Methods}

Thirty-one live-trapped males in the BRbS were used for analyses. Karyotypes were obtained from a suspension of bone marrow cells, following Ford [24]. Metaphase chromosome spreads were stained by a G-banding method [25]. Chromosome identification was performed following the Committee on Standardized Genetic Nomenclature for mice [26]. The left caudate epididyme from 13 St and $18 \mathrm{Rb}$ house mice was dissected and disaggregated in $5 \mathrm{ml}$ of phosphate buffer (PB) $0.1 \mathrm{M}$ at room temperature. After homogenization, $1 \mathrm{ml}$ of sperm solution was filtered through a nucleopore membrane $(0.2 \mu \mathrm{m})$ and fixed in $2.5 \%$ glutaraldehyde, $2 \%$ paraformaldehyde and PB 0.1 M solution. Samples were then rinsed in PB $0.1 \mathrm{M}$, postfixed in $1 \%$ osmium tetraoxide, rinsed in $\mathrm{PB}$ $0.1 \mathrm{M}$, dehydrated in graded series of ethanol and dried by the critical-point method. Membranes were observed in an S-570 scanning electron microscope (SEM; Hitachi Ltd.) at an accelerating voltage of $15 \mathrm{kV}$. From each individual, an average of 20 sperm heads in a horizontal plane, with the hook orientated to the left side and without evident structural alterations were randomly captured (Figure 1).

To determine the form of the sperm heads, sixteen landmarks and three semilandmarks were digitized using the tpsDig2 software [27] (Figure 1). The criteria used for the landmark assignation were the following: (1) top of the hook, (2) point where the hook and the upper ventral spur overlap, (3) prominence in the axis of the upper ventral spur, (4 and 7) top of the upper and lower ventral spurs, (5 and 6) inner distance between the ventral spurs, (8-11) insertion edge of the sperm head with flagellum, (12 and 13) terminal edges of the postacrosomal sheath, (14,15 and 19) basal and apical 
ridge of the equatorial crest. The semilandmarks (points 16-18) were digitized as equidistant points by the tpsDig2 'resample curve by length' option. Measurement error is an important source of variation affecting morphometric data that can increase the likelihood of type II errors and lead to biased results [28,29]. In order to evaluate the impact of measurement error in the current set of landmarks around the sperm head, in a subsample of 40 images all landmarks were digitized three times. Geometric morphometrics and modularity analyses were performed using the routines implemented by MorphoJ software [30]. Shape variation in the landmark configurations was obtained by the full Procrustes fit and the orthogonal projection to the tangent space [31]. Size was defined as centroid size (CS) [32]. In the replicated subsample, a Procrustes ANOVA comparing variation among and within sperm heads was performed to obtain the measurement error associated with landmark location [33,34]. Given that variation between sperm heads clearly exceeded that of measurement error (see Results) subsequent analyses were based on a single digitization of landmarks per head. Shape allometry, the scaling of shape with size, may conceal the patterns of modularity [35]; thus, the dependence of shape on size was calculated by means of a linear multivariate regression of the Procrustes coordinates onto the logarithm of CS [35]. Statistical significance was obtained using a permutation test with 10,000 iterations under the null hypothesis of size and shape independence [36]. The residuals obtained in the multivariate regression analyses were used for subsequent analyses [35]. First, principal component analysis (PCA) was performed with the covariance matrix of the residuals. Then, the division of the sperm head into three different sets of morphological modules was tested (Figure 1). To measure the covariation between the hypothesized sets of landmarks, the $R V$ coefficient or the multi-set $R V$ coefficient was obtained [35]. To test for modularity, this value was compared with the distribution of $R V$ values of all the alternative partitions of spatially contiguous subsets of landmarks (adjacency graphs in Figure 1) containing the same number of landmarks as the hypothesized partitions and with 10,000 random partitions [35]. Finally, we used a two-block partial least square (2B-PLS) to examine covariation between the detected modules $[37,38]$. Because of differences in mice karyotypes, these analyses were performed separating the sample into two different chromosomal groups: i) St, sperms produced by animals with 40 chromosomes and ii) $\mathrm{Rb}$, sperms from animals ranging from 30 to 39 chromosomes.

Permission to capture was granted by the Departament de Medi Ambient of the Generaltitat de Catalunya (Spain). Animals were handled in compliance with the guidelines and ethical approval by the Comissió d'Ètica en l'Experimentación Animal y Humana (CEEAH) of the Universitat Autònoma de Barcelona and by the Departament d'Agricultura, Ramaderia, Pesca, Alimentació i Medi Natural (Direcció General de Medi Natural i Biodiversitat) of the Generalitat de Catalunya (reference of the experimental procedure authorization: DAAM 6328).

\section{Competing interests}

The authors declare that they have no competing interests.

\section{Authors' contributions}

NM carried out the fieldwork, the karyotype of the animals and data acquisition. She also has participated to the design of the study, analysis and interpretation of data, and drafting the manuscript. FM conceived the study, carried out the statistical analysis, interpretation of data, and drafted the manuscript. MJL and JV participated in the design of the study and revised the manuscript. JV coordinated the study and the research project in which it is included. All authors read and approved the final manuscript.

\section{Acknowledgements}

We are indebted to J. Martínez and A. Sánchez-Chardi for their help in processing the samples by SEM and Michael Maudsley of the Serveis Lingüístics (University of Barcelona) for revising the English. This study was funded by the Spanish Ministerio de Economía y Competitividad (project number CGL2010-15243) and by a PIF grant from the Universitat Autònoma de Barcelona to NM.

\section{Author details}

${ }^{1}$ Departament de Biologia Animal, Biologia Vegetal i Ecologia, Facultat de Biociències, Universitat Autònoma de Barcelona, Cerdanyola del Vallès 08193, Spain. ²Departament de Biologia Animal, IRBio, Institut de Recerca de Biodiversitat, Facultat de Biologia, Universitat de Barcelona, Avgda. Diagonal, 643, Barcelona 08028, Spain

Received: 1 March 2013 Accepted: 21 August 2013

Published: 3 September 2013

\section{References}

1. Wagner GP, Pavlicev M, Cheverud JM: The road to modularity. Nature Rev Genet 2007, 8:921-931.

2. Klingenberg CP: Morphological integration and developmental modularity. Annu Rev Ecol Evol Syst 2008, 39:115-32.

3. Schlosser G, Wagner GP: Introduction: the modularity concept in developmental and evolutionary biology. In Modularity in development and evolution. Edited by Schlosser G, Wagner GP. Chicago: The University of Chicago Press; 2004:1-11

4. Hartwell LH, Hopfield JJ, Leibler S, Murray AW: From molecular to modular cell biology. Nature 1999, 402:C47-C52.

5. Spirin V, Mirny LA: Protein complexes and functional modules in molecular networks. Proc Nat Acad Sci 2003, 100:12123-12128.

6. Klingenberg CP: Evolution and development of shape: integrating quantitative approaches. Nature Rev Genet 2010, 11:623-635.

7. Breed WG: The spermatozoon of Eurasian murine rodents: its morphological diversity and evolution. J Morphol 2004, 261:52-69.

8. Selvaraj $V$, et al: Segregation of micron-scale membrane sub-domains in live murine sperm. J Cell Physiol 2006, 206:636-646.

9. Selvaraj V, Asano A, Buttke DE, Sengupta P, Weiss RS, Travis AJ: Mechanisms underlying the micron-scale segregation of sterols and GM1 in live mammalian sperm. J Cell Physiol 2009, 218:522-536.

10. Oko R, Clermont $Y$ : Isolation, structure and protein composition of the perforatorium of rat spermatozoa. Biol Reprod 1988, 39:673-687.

11. Oko R, Moussakova L, Clermont Y: Regional differences in composition of the perforatorium and outer periacrosomal layer of the rat spermatozoon as revealed by immunocytochemistry. Am J Anat 1990, 188:64-73.

12. Medarde N, Martínez J, Sánchez-Chardi A, López-Fuster MJ, Ventura J: Effect of Robertsonian translocations on sperm head form in the house mouse. Biol J Linn Soc. In press.

13. Hausser J, Fedyk S, Fredga K, Searle JB, Volobouev V, Wójcik JM, Zima J: Definition and nomenclature of the chromosome races of Sorex araneus. Folia Zool 1994, 43:1-9. 
14. Gündüz I, López-Fuster MJ, Ventura J, Searle JB: Clinal analysis of a chromosomal hybrid zone in the house mouse. Genet Res 2001, 77:41-51.

15. Sans-Fuentes MA, Muñoz-Muñoz F, Ventura J, López-Fuster MJ: Rb(7.17), a rare Robertsonian fusion in wild populations of the house mouse. Genet Res 2007, 89:207-213.

16. Medarde N, López-Fuster MJ, Muñoz-Muñoz F, Ventura J: Spatio-temporal variation in the structure of a chromosomal polymorphism zone in the house mouse. Heredity 2012, 109:78-89.

17. Muñoz-Muñoz F, Sans-Fuentes MA, López-Fuster MJ, Ventura J: Evolutionary modularity of the mouse mandible: dissecting the effect of chromosomal reorganizations and isolation by distance in a Robertsonian system of Mus musculus domesticus. J Evol Biol 2011, 24:1763-1776.

18. Savriama Y, Neustupa J, Klingenberg CP: Geometric morphometrics of symmetry and allometry in Micrasterias rotata (Zygnemophyceae, Viridiplantae). Beih Nova Hedw 2010, 136:43-54.

19. Needleman DJ: Cellular allometry: the spindle in development and inheritance. Curr Biol 2009, 19:846-847.

20. Korley R, Pouresmaeili F, Oko R: Analysis of the protein composition of the mouse sperm perinuclear theca and characterization of its major protein constituent. Biol Reprod 1997, 57:1426-32.

21. Toshimori $\mathrm{K}$, Ito $\mathrm{C}$ : Formation and organization of the mammalian sperm head. Arch Hystol Cytol 2003, 66:383-396.

22. Alibert P, Auffray JC: Genomic coadaptation, outbreeding depression, and, developmental stability. In Developmental stability. Causes and consequences. Edited by Polak M. New York: Oxford University Press; 2003:116-134.

23. Sans-Fuentes MA, Ventura J, López-Fuster MJ, Corti M: Morphological variation in house mice from the Robertsonian polymorphism area of Barcelona. Biol J Lin Soc 2009, 97:555-570.

24. Ford CE: The use of chromosomes markers. In Tissue Grafting and Radiation. Edited by Micklem HS, Loutit JF. New York: Academic Press; 1966:197-206.

25. Mandahl N: Methods in solid tumor cytogenetics. In Human Cytogenetics, a practical approach, Volume II. Edited by Rooney DE, Czepulkowski BH. London: IRL Press; 1992:155-187.

26. Committee on Standardized Genetic Nomenclature for Mice: Standard karyotype of the mouse Mus musculus. J Hered 1972, 63:69-72.

27. Rohlf FJ: TpsDig2, version 2.16. New York: State University of New York; 2010. Available at http://life.bio.sunysb.edu/morph/.

28. Bailey RC, Byrnes J: A new, old method for assessing measurement error in both univariate and multivariate morphometric studies. Syst Zool 1990, 39:124-130.

29. Arnqvist G, Martensson T: Measurement error in geometric morphometrics: empirical strategies to assess and reduce its impact on measures of shape. Acta Zool Hung 1998, 44:73-96.

30. Klingenberg CP: MorphoJ: an integrated software package for geometric morphometrics. Mol Ecol Resources 2011, 11:353-357.

31. Dryden IL, Mardia KV: Statistical shape analysis. Chichester: John Wiley; 1998.

32. Bookstein FL: Morphometric tools for landmark data. 1st edition. Cambridge: Cambridge Univ Press; 1991.

33. Klingenberg CP, Mclntyre GS: Geometric morphometrics of developmental instability: analyzing patterns of fluctuating asymmetry with Procrustes methods. Evolution 1998, 52:1363-1375.

34. Klingenberg CP, Barluenga M, Meyer A: Shape analysis of symmetric structures: quantifying variation among individuals and asymmetry. Evolution 2002, 56:1909-1920.

35. Klingenberg CP: Morphometric integration and modularity in configurations of landmarks: tools for evaluating a priori hypotheses. Evol Dev 2009, 11:405-421.

36. Good P: Permutation tests: a practical guide to resampling methods for testing hypotheses. New York: Springer-Verlag; 1994

37. Rohlf FJ, Corti M: The use of two-block partial least-squares to study covariation in shape. Syst Biol 2000, 49:740-753.

38. Singh N, Harvati K, Hublin JJ, Klingenberg CP: Morphological evolution through integration: a quantitative study of cranial integration in Homo, Pan, Gorilla and Pongo. J Hum Evol 2012, 62:155-164.

doi:10.1186/1471-2148-13-179

Cite this article as: Medarde et al:: Variational modularity at the cell level: insights from the sperm head of the house mouse. $B M C$ Evolutionary Biology 2013 13:179.

\section{Submit your next manuscript to BioMed Central and take full advantage of:}

- Convenient online submission

- Thorough peer review

- No space constraints or color figure charges

- Immediate publication on acceptance

- Inclusion in PubMed, CAS, Scopus and Google Scholar

- Research which is freely available for redistribution 